\title{
Addition of atezolizumab to nab-paclitaxel plus carboplatin is a new standard option for the first-line treatment for non-squamous non-small cell lung cancer
}

\author{
Teppei Yamaguchi, Toyoaki Hida \\ Department of Thoracic Oncology, Aichi Cancer Center Hospital, Nagoya, Aichi, Japan \\ Correspondence to: Teppei Yamaguchi. Department of Thoracic Oncology, Aichi Cancer Center Hospital, 1-1, Kanokoden, Chikusa-ku, Nagoya, Aichi \\ 464-8681, Japan. Email: yteppei@aichi-cc.jp. \\ Comment on: West H, McCleod M, Hussein M, et al. Atezolizumab in combination with carboplatin plus nab-paclitaxel chemotherapy compared with \\ chemotherapy alone as first-line treatment for metastatic non-squamous non-small-cell lung cancer (IMpower130): a multicentre, randomised, open- \\ label, phase 3 trial. Lancet Oncol 2019;20:924-37.
}

Submitted Nov 27, 2019. Accepted for publication Dec 13, 2019.

doi: $10.21037 /$ tcr.2019.12.57

View this article at: http://dx.doi.org/10.21037/tcr.2019.12.57

For over a decade, the discovery of driver oncogenes and the development of specific targeted drugs have led to significant advances in the treatment for metastatic nonsmall cell lung cancer (NSCLC). Molecular targeted drugs targeting EGFR, ALK, ROS1, and BRAF have been developed, and each advance has resulted in improved outcomes in NSCLC. Meanwhile, in the absence of molecular targeted drugs, patients have frequently received platinum combination therapy, which is associated with progression-free survival (PFS) and overall survival (OS) of 4-6 and 9-12 months, respectively $(1,2)$.

Immune checkpoint inhibitors (ICIs) targeting the programmed death-1 (PD-1)/programmed deathligand-1 (PD-L1) have dramatically transformed the lung cancer treatment paradigm. The PD-1 inhibitor nivolumab facilitated the use of immunotherapy in NSCLC by producing improved OS rates over docetaxel for previously treated squamous and non-squamous NSCLC in the CheckMate 017 and CheckMate 057 trials $(3,4)$. Subsequently, pembrolizumab monotherapy has been used in patients with high tumor PD-L1 levels, as indicated by a tumor proportion score (TPS) of $50 \%$ or greater, based on its better efficacy than platinum doublet therapy in the firstline setting. Recently, combining PD-1/PD-L1 inhibitors with platinum doublet treatment has resulted in prolonged both OS and PFS in patients with previously untreated advanced NSCLC. Additionally, most of these patients did not have driver mutations. Therefore, combination therapy with ICIs and chemotherapy has been the most common treatment strategy for malignancies for which no molecular targeted therapy is available.

The randomized, phase III, open-label, global, multicenter IMpower130 trial investigated the efficacy and safety of adding atezolizumab to nab-paclitaxel and carboplatin in patients with previously untreated metastatic non-squamous NSCLC (5). In this trial, 724 participants were randomly assigned in a 2:1 ratio to treatment with atezolizumab plus nab-paclitaxel and carboplatin or nabpaclitaxel and carboplatin alone. Induction treatments, consisting of atezolizumab together with carboplatin and nab-paclitaxel or chemotherapy alone, were administered in four or six 3-week cycles. Afterward, the atezolizumab combination therapy group was continually administered atezolizumab monotherapy as a maintenance treatment, whereas the chemotherapy alone group received either switch maintenance pemetrexed or best supportive care. This study met both co-primary endpoints demonstrating superiority of PFS and OS versus chemotherapy in the intention-to-treat wild-type patients. Specifically, significant improvements of PFS and OS were observed in the atezolizumab combination therapy group versus the chemotherapy alone group [median PFS: 7.0 vs. 5.5 months; hazard ratio (HR): $0.64 ; 95 \%$ confidence interval (CI): 0.54-0.77; $\mathrm{P}<0.0001$; median OS: 18.6 vs. 13.9 months; HR: 0.79; $95 \%$ CI: $0.64-0.98 ; \mathrm{P}=0.033$ ).

Favorable survival benefits were observed for the 
atezolizumab plus chemotherapy arm in almost all subgroups in the IMpower130 trial. However, there was no apparent benefit in patients with liver metastases, EGFR mutation, or $A L K$ rearrangement (5). In the IMpower150 trial, the strategy of adding atezolizumab to paclitaxel/ carboplatin/bevacizumab produced good survival outcomes in patients with liver metastases (6). Going back in time, in the E4599 trial, the efficacy of adding bevacizumab to paclitaxel plus carboplatin in NSCLC patients with liver metastases was indicated (2). Whereas, in the IMpower130 trial, no additional effect was noted in patients with liver metastases, which may be because the study treatment did not include bevacizumab.

In the IMpower130 trial, the analysis of patients with $A L K$ rearrangement or EGFR mutation was performed in an extremely small population. The atezolizumab combination therapy arm included $32(7 \%)$ patients, and the chemotherapy alone arm included $12(5 \%)$ patients. In patients with $A L K$ rearrangement or EGFR mutation, the median PFS and OS were 7.0 (HR: 0.75; 95\% CI: $0.36-1.54$ ) and 14.4 months (HR: 0.98 ; 95\% CI: 0.41-2.31), respectively, in the atezolizumab combination therapy group, versus 6.0 and 10.0 months, respectively, in the chemotherapy alone group.

The IMpower150 trial is the only study to reveal a clinical benefit of atezolizumab plus chemotherapy in patients with $A L K$ rearrangement- or EGFR mutationpositive NSCLC (6). However, this trial included small numbers of patients with $A L K$ rearrangement or EGFR mutation, including 44 patients in the atezolizumab plus paclitaxel/carboplatin/bevacizumab group and 64 patients in the paclitaxel/carboplatin/bevacizumab group (6). Data on the combination of PD-1 axis inhibitors and chemotherapy for patients with $E G F R / A L K$-positive tumor remain insufficient. To resolve this problem, we have to await the results of the CheckMate 722 and KEYNOTE-789 trials, which are ongoing phase III studies exploring the role of adding ICI to chemotherapy in patients with TKI-resistant, EGFR-positive NSCLC.

According to the results of the IMpower130 trial, the addition of atezolizumab to nab-paclitaxel plus carboplatin has become a novel standard of care in the first-line setting for advanced non-squamous NSCLC. How should clinicians choose from several available PD-1/PD-L1 inhibitors and chemotherapy combination therapy in clinical practice? To date, there is no evidence to answer this question because there has been no comparative study between ICI chemotherapy combinations. Therefore, the characteristics of each drug may be helpful in selecting an appropriate treatment.

The first issue is whether the antitumor effects of PD-1 and PD-L1 inhibitors are similar. Regarding the effect of ICIs alone, OS was successfully extended in two trials that explored the efficacy of pembrolizumab monotherapy in the first-line setting, including a study of patients with a PD-L1 TPS $\geq 50 \%$ (KEYNOTE-024) and a second trial of patients with a PD-L1 TPS $\geq 1 \%$ (KEYNOTE-042) $(7,8)$. In addition, data reported from the phase III IMpower110 trial at the ESMO Congress 2019 indicated that atezolizumab monotherapy prolonged survival in people with metastatic NSCLC and high PD-L1 levels compared with the effects of chemotherapy alone (9). Meanwhile, in a meta-analysis comparing the clinical benefits of anti-PD-1 and antiPD-L1 antibodies in NSCLC patients who failed first-line chemotherapy, the response rate to anti-PD-1 antibodies tended to be better than that to anti-PD-L1 antibodies (10). However, in another meta-analysis, patients who treated with anti-PD-1 antibodies had increased rates of all-grade and high-grade immune-related adverse events (irAEs) than those who received anti-PD-L1 inhibitors (11). Therefore, the anti-PD-L1 antibody atezolizumab may have an inferior response rate to anti-PD-1 antibodies, although its incidence of irAEs may be lower.

The second issue is proper selection of the combination chemotherapy. Pemetrexed, which was used in the KEYNOTE-189 trial, has lower incidences of peripheral neuropathy, arthralgia, and alopecia than paclitaxel/nabpaclitaxel, which could be associated with patient quality of life (12). Therefore, pemetrexed is an easy-to-use agent from the viewpoint of side effect management. However, pemetrexed is mainly excreted by the kidneys, and it is not recommended for patients with impaired renal function (creatinine clearance rates $<45 \mathrm{~mL} / \mathrm{min}$ ) because of increased myelosuppression $(13,14)$. Contrarily, paclitaxel is metabolized mainly in the liver, with less than $10 \%$ of the dose excreted by the kidneys (15). Therefore, paclitaxel/ nab-paclitaxel is generally feasible in patients with renal impairment $(16,17)$. In the phase III CA031 trial, nabpaclitaxel/carboplatin demonstrated superiority in OS versus paclitaxel/carboplatin among patients $\geq 70$ years old subset (18). Although, the proportions of elderly people ( $\geq 75$ years old) in the IMpower 130 trial were not high (11\% in the atezolizumab combination therapy arm and $14 \%$ in the chemotherapy alone arm), nab-paclitaxel plus carboplatin treatment may potentially be suitable for elderly patients considering the results of the CA031 trial. 
If pemetrexed is not considered appropriate, then treatment including paclitaxel/nab-paclitaxel may be selected. The phase III PointBreak trial compared the safety and efficacy of pemetrexed/carboplatin/bevacizumab followed by maintenance pemetrexed/bevacizumab with those of paclitaxel/carboplatin/bevacizumab followed by maintenance bevacizumab in metastatic NSCLC patients with non-squamous cell carcinoma histology. In this trial, survival benefits were more likely to be obtained in the paclitaxel/carboplatin/bevacizumab arm than in the pemetrexed/carboplatin/bevacizumab arm when the tissue type was classified as "other or indeterminant" rather than adenocarcinoma or large cell (19). A similar tendency was observed in the PRONOUNCE study, which compared the safety and efficacy of pemetrexed/carboplatin followed by maintenance pemetrexed with those of paclitaxel/ carboplatin/bevacizumab followed by maintenance bevacizumab (20). Therefore, in the case of non-squamous/ non-adenocarcinoma/non-large cell NSCLC, the IMpower130/IMpower150 regimens containing paclitaxel/ nab-paclitaxel may be more effective.

We welcome the addition of atezolizumab to nabpaclitaxel plus carboplatin, representing a new treatment option for non-squamous NSCLC. Furthermore, through the development of combination strategies centering ICIs based on precise biomarkers, it is expected that more effective immunotherapy combinations will be developed for individual patients.

\section{Acknowledgments}

We thank Joe Barber Jr., PhD, from Edanz Group (https:// en-author-services.edanzgroup.com/) for editing a draft of this manuscript.

Funding: None.

\section{Footnote}

Provenance and Peer Review: This article was commissioned by the Editorial Office, Translational Cancer Research. It has not been sent for external peer review.

Conflicts of Interest: All authors have completed the ICMJE uniform disclosure form (available at http://dx.doi.org/10.21037/ tcr.2019.12.57). Dr. Hida has obtained research grants from Ono Pharmaceutical, Novartis Pharma, Chugai Pharmaceutical, Eli Lilly, Taiho Pharmaceutical, AstraZeneca, Nippon Boehringer Ingelheim, Pfizer, Bristol-Meyers Squibb,
Clovis Oncology, Eisai, Takeda Bio, Sumitomo Dainippon Pharma, AbbVie, Merck Serono, MSD, Kyowa Hakko Kirin, Daiichi Sankyo, and Astellas and has received personal fees from Ono Pharmaceutical, Novartis Pharma, Chugai Pharmaceutical, Eli Lilly, Taiho Pharmaceutical, AstraZeneca, Nippon Boehringer Ingelheim, Pfizer, Clovis Oncology, and Bristol-Myers Squibb. Dr. Yamaguchi has no conflicts of interest to declare.

Ethical Statement: The authors are accountable for all aspects of the work in ensuring that questions related to the accuracy or integrity of any part of the work are appropriately investigated and resolved.

Open Access Statement: This is an Open Access article distributed in accordance with the Creative Commons Attribution-NonCommercial-NoDerivs 4.0 International License (CC BY-NC-ND 4.0), which permits the noncommercial replication and distribution of the article with the strict proviso that no changes or edits are made and the original work is properly cited (including links to both the formal publication through the relevant DOI and the license). See: https://creativecommons.org/licenses/by-nc-nd/4.0/.

\section{References}

1. Scagliotti GV, Parikh P, von Pawel J, et al. Phase III study comparing cisplatin plus gemcitabine with cisplatin plus pemetrexed in chemotherapy-naive patients with advanced-stage non-small-cell lung cancer. J Clin Oncol 2008;26:3543-51.

2. Sandler A, Gray R, Perry MC, et al. Paclitaxel-carboplatin alone or with bevacizumab for non-small-cell lung cancer. N Engl J Med 2006;355:2542-50.

3. Brahmer J, Reckamp KL, Baas P, et al. Nivolumab versus Docetaxel in Advanced Squamous-Cell Non-Small-Cell Lung Cancer. N Engl J Med 2015;373:123-35.

4. Borghaei H, Paz-Ares L, Horn L, et al. Nivolumab versus Docetaxel in Advanced Nonsquamous Non-Small-Cell Lung Cancer. N Engl J Med 2015;373:1627-39.

5. West H, McCleod M, Hussein M, et al. Atezolizumab in combination with carboplatin plus nab-paclitaxel chemotherapy compared with chemotherapy alone as firstline treatment for metastatic non-squamous non-small-cell lung cancer (IMpower130): A multicentre, randomised, open-label, phase 3 trial. Lancet Oncol 2019;20:924-37.

6. Socinski MA, Jotte RM, Cappuzzo F, et al. Atezolizumab for First-Line Treatment of Metastatic Nonsquamous 
NSCLC. N Engl J Med 2018;378:2288-2301.

7. Reck M, Rodríguez-Abreu D, Robinson AG, et al. Pembrolizumab versus Chemotherapy for PD-L1Positive Non-Small-Cell Lung Cancer. N Engl J Med 2016;375:1823-33.

8. Mok TSK, Wu YL, Kudaba I, et al. Pembrolizumab versus chemotherapy for previously untreated, PD-L1expressing, locally advanced or metastatic non-small-cell lung cancer (KEYNOTE-042): A randomised, open-label, controlled, phase 3 trial. Lancet 2019;393:1819-30.

9. Spigel D, de Marinis F, Giaccone G, et al. IMpower110: Interim overall survival (OS) analysis of a phase III study of atezolizumab (atezo) vs platinum-based chemotherapy (chemo) as first-line (1L) treatment (tx) in PD-L1-selected NSCLC. Ann Oncol 2019;30:v851-v934.

10. You W, Liu M, Miao JD, et al. A Network Meta-analysis Comparing the Efficacy and Safety of Anti-PD-1 with Anti-PD-L1 in Non-small Cell Lung Cancer. J Cancer 2018;9:1200-06.

11. Sun X, Roudi R, Dai T, et al. Immune-related adverse events associated with programmed cell death protein-1 and programmed cell death ligand 1 inhibitors for nonsmall cell lung cancer: A PRISMA systematic review and meta-analysis. BMC Cancer 2019;19:558.

12. Gandhi L, Rodríguez-Abreu D, Gadgeel S, et al. Pembrolizumab plus Chemotherapy in Metastatic NonSmall-Cell Lung Cancer. N Engl J Med 2018;378:2078-92.

13. Mita AC, Sweeney CJ, Baker SD, et al. Phase I and pharmacokinetic study of pemetrexed administered every 3 weeks to advanced cancer patients with normal and impaired renal function. J Clin Oncol 2006;24:552-62.

14. Ando Y, Hayashi T, Ujita M, et al. Effect of renal function on pemetrexed-induced haematotoxicity. Cancer
Chemother Pharmacol 2016;78:183-9.

15. Wiernik PH, Schwartz EL, Strauman JJ, et al. Phase I clinical and pharmacokinetic study of taxol. Cancer Res 1987;47:2486-93.

16. Kawate S, Takeyoshi I, Morishita Y. Pharmacokinetics of paclitaxel in a hemodialysis patient with advanced gastric cancer: A case report. World J Gastroenterol 2006;12:5237-9.

17. Langer CJ, Hirsh V, Ko A, et al. Weekly nab-paclitaxel in combination with carboplatin as first-line therapy in patients with advanced non-small-cell lung cancer: Analysis of safety and efficacy in patients with renal impairment. Clin Lung Cancer 2015;16:112-20.

18. Socinski MA, Bondarenko I, Karaseva NA, et al. Weekly nab-paclitaxel in combination with carboplatin versus solvent-based paclitaxel plus carboplatin as first-line therapy in patients with advanced non-small-cell lung cancer: final results of a phase III trial. J Clin Oncol 2012;30:2055-62.

19. Patel JD, Socinski MA, Garon EB, et al. Point Break: A randomized phase III study of pemetrexed plus carboplatin and bevacizumab followed by maintenance pemetrexed and bevacizumab versus paclitaxel plus carboplatin and bevacizumab followed by maintenance bevacizumab in patients with stage IIIB or IV nonsquamous non-small-cell lung cancer. J Clin Oncol 2013;31:4349-57.

20. Zinner RG, Obasaju CK, Spigel DR, et al. PRONOUNCE: randomized, open-label, phase III study of first-line pemetrexed + carboplatin followed by maintenance pemetrexed versus paclitaxel + carboplatin + bevacizumab followed by maintenance bevacizumab in patients ith advanced nonsquamous non-small-cell lung cancer. J Thorac Oncol 2015;10:134-42.
Cite this article as: Yamaguchi T, Hida T. Addition of atezolizumab to nab-paclitaxel plus carboplatin is a new standard option for the first-line treatment for non-squamous non-small cell lung cancer. Transl Cancer Res 2019;8(8):E11E14. doi: 10.21037/tcr.2019.12.57 